\title{
Méthodologie de la cartographie des risques naturels liés
}

\section{aux mouvements de terrain}

\section{A. FARES}

M. ROLLET

P. BROQUET

Laboratoire de géologie structurale et appliquée 1. place Leclerc 25000 Besançon

\author{
(Région de Taounate - Rif, Nord du Maroc)
}

Cette étude présente une méthodologie de la cartographie des risques naturels liés aux mouvements de terrain à l'échelle du $1 / 25000$, qui utilise un mode de cartographie numérique basé sur une simulation du risque en fonction de l'importance et de la typologie des instabilités de versant.

Les facteurs constituant les éléments du risque pour cette cartographie sont relatifs à la topographie, à la lithologie et à la géomorphologie. Ce choix des facteurs nous permet de délimiter le cadre propice aux déclenchements des mouvements de terrain. L'évaluation du risque résultant de l'action conjointe des facteurs a été effectuée suivant deux méthodes, la méthode arithmétique et la méthode probabiliste, aboutissant à deux cartes synthétiques dont une seule, fondée sur la méthode probabiliste servira comme carte des risques naturels; facile à lire, elle est donc utilisable par les différents décideurs de l'aménagement du territoire.

Mots clés: Cartographie, géologie, simulation, mouvement de terrain, risque, méthodologie, quantification.

\section{Methodical test of natural risks relative to ground movements cartography}

\author{
(Taounate area - Rif, North Marroco)
}

The present study suggests a methodical test of mapping the natural risks relative to ground movements at a $1 / 25000$ scale.

This methodical test riks uses a numeric cartography based on a risk simulation in accordance with the typology importance of slopes instabilities.

The factors making the risk elements selected in this cartography are those of topography, lithology and geomorphology. This selection enable us to delimit the favourable frame releasing ground movements. The risk evaluation resulting from the selected factors united actions is realised using two methods. The first one is arithmetic whereas the second is probabilistic.

Theese two methods lead to two synthetic maps, the one using the probabilistic method will be employed to realise a natural risks map, being easy to read and helpful for different users in urbanism.

Key words : Cartography, geology, simulation, ground movements, risk, methodical test, quantification. 


\section{Introduction}

Le Rif forme un domaine d'étude riche et varié des instabilités de versants. Ces mouvements de terrain, au niveau du site de Taounate (Rif central), gênent l'aménagement de la ville du même nom et constituent un risque, d'où l'intérêt d'une cartographie à caractêre prévisionnelle pour définir les sites les plus exposés aux instabilités des versants.

Historiquement, la cartographie des risques naturels a commencé pour la première fois au Maroc dans les années soixante, en même temps que les cartes géotechniques de Casablanca, de Fès, de Tanger... En 1968, A. Millies-Lacroix établit pour l'ensemble du Rif une carte prévisionnelle des mouvements de terrain à une échelle de 1/1000000. Les facteurs utilisés dans cette cartographie sont la lithologie au sens large, les hauteurs moyennes de précipitation annuelle, la topographie, le couvert végétal et l'action anthropique. La région de Taounate apparait dans des zones comprises entre les degrés 6 et 9, correspondant aux susceptibilités maximales de la carte prévisionnelle.

En France, les études de cartographie des risques naturels ont commencé à partir du début des années soixante-dix, par le Bureau de Recherche Géologiques et Minières (BRGM) et le laboratoire de géologie appliquée de I'Université de Grenoble, dans une cartographie commune intitulée Plan Zermos «zones exposées aux risques des mouvements du sol et du sous sol ») (W. Chazan, 1973). Les objectifs de ce programme n'ont pas été totalement atteints (P. Antoine, 1977), puisque la conception du risque reste une notion subjective interprétée différemment selon les auteurs des cartes Zermos réalisées jusqu'à maintenant.

Nous avons repris les préoccupations de la cartographie Zermos et défini une méthodologie en utilisant le mode de cartographie numérique basée sur la simulation des facteurs à partir des observations et des données de surface. Notre méthode s'appuie sur des moyens faciles à mettre en ceuvre et peu coûteux, à savoir: une carte topographique à l'échelle du 1/25000, une carte géologique levée à l'échelle du 1/25000, des photographies aériennes et quelques essais géotechniques. A partir de ces données, nous avons élaboré une série de cartes qui s'enchaînent dans un ordre logique pour aboutir à la carte des risques naturels liés aux mouvements de terrain de la région de Taounate.

\section{2}

\section{Les facteurs déterminant la genèse des mouvements de terrain}

La genèse d'un mouvement de terrain résulte de l'action simultanée de plusieurs facteurs. Certains sont permanents et forment le cadre propice créant les conditions indispensables aux mouvements, à savoir: la topographie, la lithologie et la géomorphologie; les autres dynamiques ont un rôle déclenchant et sont représentés par le climat, I'hydrologie, l'évolution de la dynamique actuelle des mouvements de terrain (A.Millies-Lacroix, 1981), l'action de l'homme, la végétation et la sismicité. Ces facteurs dynamiques ont une intensité et une fréquence variables dans le temps et dans l'espace.

Nous avons fait le choix de cartographier à l'échelle du 1/25000 les facteurs permanents afin de délimiter les secteurs qui présentent le cadre favorable aux déclenchements des mouvements de terrain.

\section{3}

\section{Établissement de la carte des risques naturels}

\section{1}

\section{Méthodologie (Fig. 1)}

Pour aboutir à une carte des secteurs susceptibles d'être le siège des mouvements de terrain, nous avons retenu les facteurs liés à la topographie, à la nature et la structure du matériau et à la géomorphologie. Ces facteurs sont cartographiés séparément pour obtenir les cartes de facteurs du risque. La superposition de ces cartes fait ressortir les relations entre la lithologie, la pente et les indices d'instabilité. Une étude basée sur les calculs de rapports de surfaces nous permet de fonder une simulation du risque que nous avons graduée en cinq niveaux dans une échelle numérique (1, 2, 3, 4 et 5). Ceci nous conduit à transcrire les cartes de facteurs en cartes numériques appelées cartes de simulation du risque. La carte de l'interprétation du risque délimite des secteurs où figurent le niveau du risque assigné à chaque facteur selon des triplets.

L'évaluation du risque résultant de l'action conjointe des facteurs a été effectuée suivant deux méthodes: la méthode arithmétique et la méthode probabiliste aboutissant à deux cartes synthétiques dont une seule servira comme carte des risques naturels à la suite de la comparaison des deux démarches.

\section{2}

\section{Les cartes de facteurs}

\section{3,81}

\section{Carte des pentes}

Nous avons établi une carte des pentes à partir de la carte topographique à l'échelle du 1/25000.Elle présente six classes de pente de [0-2,5\%], [2,5-5\%], [5$10 \%],[10-20 \%],[20-40 \%]$ et $[40-100 \%]$.

\section{2 .9}

\section{Carte des faciès lithologiques}

Elle a été établie à partir de la carte géologique à l'échelle du 1/50000 (G. Suter, 1967) et de levés nouveaux. Elle présente une notice explicative qui classe les faciès suivant les caractéristiques géologiques, hydrogéologiques et géotechniques. Nous y avons annexé également la carte de distribution de la fracturation dans les massifs gréseux qui présente en plus des rosaces de fracturation, le pendage et le degré d'hétérogénéité en fonction de la dimension des blocs délimités par les fractures. 


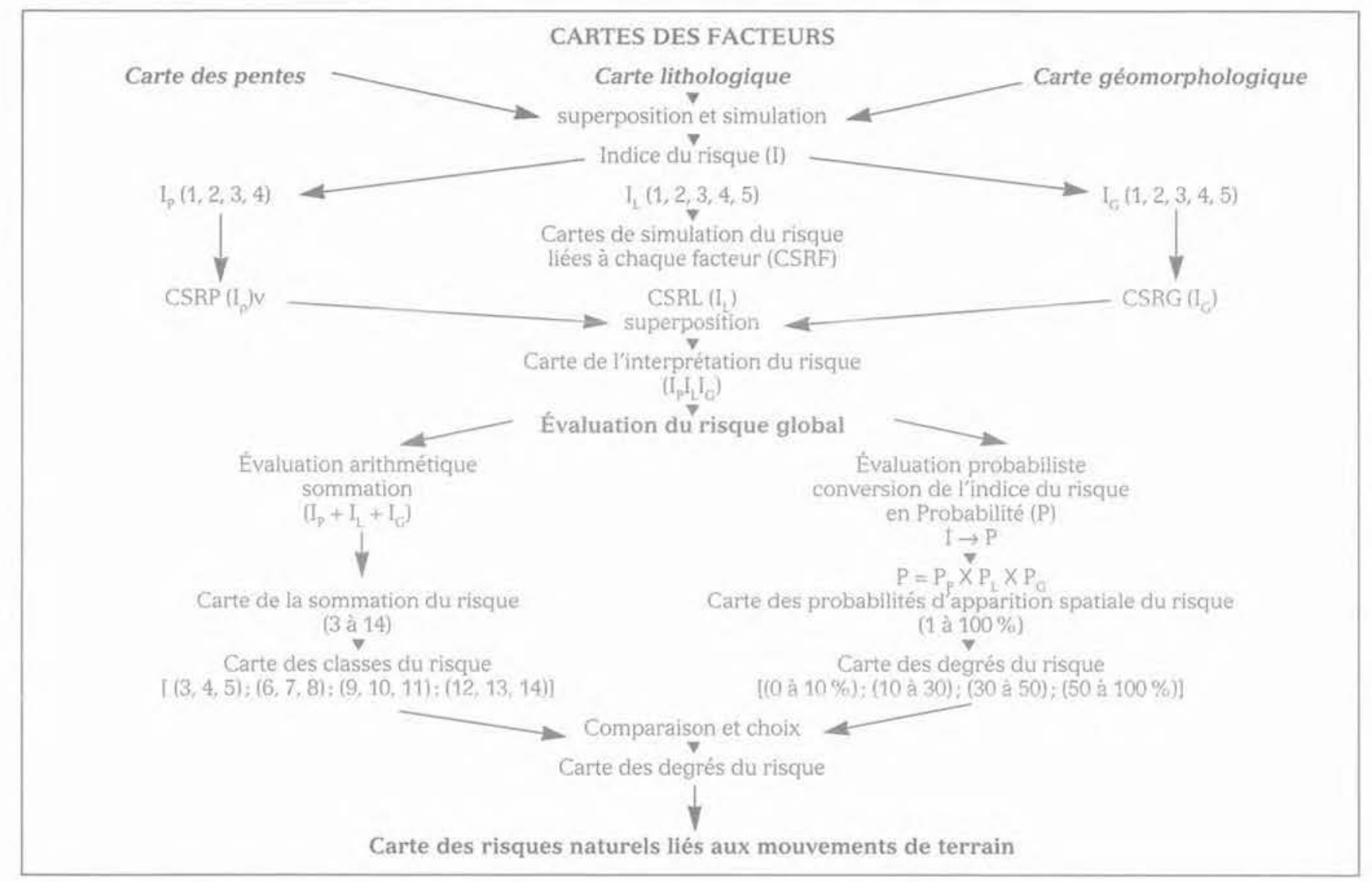

FIG.1 Essai méthodologique de la cartographie des risques naturels.

Methodical test of the natural risks cartography
(I): Indice du risque
$\left(\mathrm{I}_{\mathrm{p}}\right)$ : Indice du risque lié à la pente
$\left(I_{1}\right)$ : Indice du risque lié à la lithologie
$\left(\mathrm{I}_{\mathrm{G}}\right)$ : Indice du risque lié à la géomorphologie
(P): Probabilité du risque
$(\mathrm{PP})$ : Probabilité du risque lié à la pente
$\left(\mathrm{P}_{\mathrm{L}}\right)$ : Probabilité du risque lié à la lithologie
$\left(\mathrm{P}_{G}\right)$ : Probabilité du risque lié à la géomorphologie

TABIfauI Degré d'instabilité des classes des pentes des marnes bleues de la région de Taounate, Instability degree of blues marls slopes classes of Taounate area.

\begin{tabular}{|c|c|c|c|c|}
\hline Classe des pentes & $\begin{array}{l}\text { Surfaces totales } \\
\text { St }\left(\mathrm{km}^{2}\right)\end{array}$ & $\begin{array}{l}\text { Surfaces instables } \\
\qquad \text { Si }\left(\mathrm{km}^{2}\right)\end{array}$ & $\begin{array}{c}\text { Degré d'instabilité } \\
\text { Sì/St }(\%)\end{array}$ & Types d'instabilité \\
\hline 2,5 à $5 \%$ & 0,197 & 0 & 0 & \\
\hline 5 à $10 \%$ & 2.74 & 0,175 & 6,4 & \multirow{3}{*}{$\begin{array}{c}\text { Solifluxion } \\
\text { et } \\
\text { ravinement }\end{array}$} \\
\hline 10 à $20 \%$ & 13,35 & 2,35 & 17,6 & \\
\hline 20 à $40 \%$ & 3,45 & 1,15 & 33,4 & \\
\hline
\end{tabular}

\section{Carte des indices d'instabilité}

Elle a été établie à partir de l'étude des photographies aériennes de plusieurs missions espacées dans le temps (1951, 1960, 1979 et 1986) complétée par les observations sur le terrain concernant les dommages occasionnés. En dehors des ravinements et des badlands qui sont des phénomènes actuellement actifs, la cartographie des mouvements de masse a englobé systématiquement les formes actives où le risque est certain et les formes anciennes stabilisées ou en période de rémission provisoire.

\section{3}

\section{Les cartes analytiques}

\section{Quantification du risque}

Dans la nature, chaque faciès lithologique présente une pente limite en dessous de laquelle le versant n'engendre aucune instabilité. Nous avons procédé à une étude des surfaces basée sur la superposition sec- 
teur par secteur de la lithologie, des pentes et des indices d'instabilité; nous avons ainsi défini pour chaque faciès les degrés d'instabilité en fonction des classes de pentes (rapport des surfaces instables sur la surface totale de la classe considérée). Cette confrontation des cartes de facteurs nous permet de situer approximativement la pente limite de chaque faciès:

Les marnes bleues ne présentent pas de pente supérieure à $40 \%$ (Tab. I). A partir des pourcentages des surfaces instables, on peut caler approximativement la pente limite vers $10 \%$ et plus précisément entre 5 et $10 \%$.

Le pourcentage des surfaces instables élevé de la classe $[10,20]$ traduit I'héritage d'anciennes coulées et correspond à des terrains relativement altérés (Tab. II). La pente limite des marnes schisteuses ne pourra avoir une valeur supérieure à $20 \%$ que si la roche est saine.

Contrairement aux marnes bleues et aux marnes schisteuses, les grès n'ont pas de pente limite (Tab. III). Les instabilités correspondant aux différentes classes dépendent d'autres facteurs tels que la structure et l'altération.

Il ressort de cette confrontation, que toutes les pentes inférieures à $10 \%$ sont relativement stables et qu'á partir de $10 \%$ la stabilité des terrains dépend de la nature lithologique, de la structure ou de l'altération.

\section{2}

\section{Les cartes de simulation du risque}

A chacun des facteurs du risque, nous avons attribué un indice ( $\mathrm{I}_{\mathrm{f}}$ dans une échelle numérique en rapport avec son importance relative dans la genèse des instabilités. Cette simulation nous permet de traduire les cartes de facteurs en cartes de simulation du risque.

\section{- Carte de simulation du risque lié à la pente}

La détermination cartographique de la pente limite naturelle pour chaque matériau rend possible l'utilisation directe de la carte des pentes dans une cartographie des risques naturels. Nous pouvons comparer les pentes des versants (Pv) aux pentes limites (Pl) des différents faciès et attribuer un indice de risque $\left(1_{p}\right)$. comme suit:

- si Pv est inférieur à Pl, la pente du versant est stable et I prend la valeur minimum de: 1 ;

- sî Pv est sensiblement égal à Pl, I est égal à : 2

- si Pv est supérieur à $\mathrm{Pl}, \mathrm{I}$ est égal à : 3 ;

- si Pv est très superieur a PI, $I_{p}$ prend la valeur maximum de : 4 .

Le tableau IV montre les différents indices attribués en fonction des degrés d'instabilité des classes de pente:

TABLEAUIII Degré d'instabilité des classes des pentes des marnes schisteuses du Sénonien de la région de Taounate. Instability degree of Senonian schistous maris slopes classes of Taounate area:

\begin{tabular}{|c|c|c|c|c|}
\hline Classe des pentes & $\begin{array}{l}\text { Surfaces totales } \\
\text { St (km?) }\end{array}$ & $\begin{array}{c}\text { Surfaces instables } \\
\text { Si (km) }\end{array}$ & $\begin{array}{c}\text { Degré d'instabilité } \\
\text { Si/St (\%) }\end{array}$ & Types d'instabilité \\
\hline 5 à $10 \%$ & $0,4,33$ & 0 & 0 & \\
\hline 10 à $20 \%$ & 3,733 & 1,4124 & 37,83 & \multirow[t]{2}{*}{ Coulées anciennes } \\
\hline 20 à $40 \%$ & 3,2715 & 1,3185 & 40,3 & \\
\hline$>$ à $40 \%$ & 0,5308 & 0.2455 & 46,25 & Niches de départ et ravinement \\
\hline
\end{tabular}

TABtEAUIII Degré d'instabilité des classes des pentes des grès du Tortonien supérieur de la région de Taounate. Instability degree of Upper Tortonian sandstones slopes classes of Taounate area.

\begin{tabular}{|c|c|c|c|c|}
\hline Classe des pentes & $\begin{array}{c}\text { Surfaces totales } \\
\text { St }\left(\mathrm{km}^{2}\right)\end{array}$ & $\begin{array}{l}\text { Surfaces instables } \\
\text { Si }\left(\mathrm{km}^{2}\right)\end{array}$ & $\begin{array}{c}\text { Degré d'instabilité } \\
\text { Si/St }(\%)\end{array}$ & Types d'instabilité \\
\hline 5 à $10 \%$ & 0,2314 & 0 & 0 & \\
\hline 10 से $20 \%$ & 2,5422 & 0,4453 & 17,5 & \multirow[t]{2}{*}{ Glissement de terrain } \\
\hline 20 à $40 \%$ & 7,9492 & 0.4242 & 5,4 & \\
\hline$>$ à $40 \%$ & 1,6726 & 0,1403 & 8,38 & Glissement et éboulement \\
\hline
\end{tabular}

TAalEAUIV Indices de risque attribués aux différentes classes de pentes en fonction de Ia lithologie. Risk indices attributed to different siopes classes in accordance with the lithology.

\begin{tabular}{|c|c|c|c|c|c|c|c|}
\hline $\begin{array}{c}\text { Classe } \\
\text { de pentes }\end{array}$ & $\begin{array}{l}\text { Terrasses } \\
\text { anciennes }\end{array}$ & $\begin{array}{c}\text { Marnes } \\
\text { bleues }\end{array}$ & Molasses & Grès & $\begin{array}{c}\text { Marnes } \\
\text { schisteuses }\end{array}$ & $\begin{array}{l}\text { Schistes } \\
\text { gréseux }\end{array}$ & $\begin{array}{l}\text { Complexe } \\
\text { bréchique }\end{array}$ \\
\hline 2,5 à $5 \%$ & 1 & 1 & $\theta$ & $(-)$ & $(-)$ & $(-)$ & $(-)$ \\
\hline 5 à $10 \%$ & $(-)$ & 2 & $(-)$ & 1 & 1 & $(-)$ & $H$ \\
\hline $10 a ̀ 20 \%$ & $H$ & 3 & 2 & 2 & 2 & $(-)$ & $(-)$ \\
\hline $20 a \dot{4} 40 \%$ & $(-)$ & 4 & 3 & 3 & 3 & 1 & 3 \\
\hline 40 à $100 \%$ & $(-)$ & $(-)$ & 4 & 4 & 4 & 2 & $(-)$ \\
\hline
\end{tabular}




\section{- Carte de simulation du risque lié à la lithologie}

Nous pouvons faire une première approximation en comparant le degré d'instabilité des différents faciès lithologiques (Tab. V).

Compte tenu du degré et du type d'instabilité, de la nature et de l'épaisseur de la couverture d'altération, et de la structure; nous avons traduit la carte des faciès lithologiques en une carte de simulation du risque lié à la lithologie, basée sur les indices du risque attribués aux faciès suivants:

- les molasses : 5 ;

- les marnes bleues saines et altérées, le complexe bréchique gypsifère, les marnes schisteuses altérées, les grès très fracturés à pendage conforme à la pente, les grès à couverture d'altération issue d'un mouvement de masse: 4 ;

- les marnes schisteuses, les grès à pendage conforme à la pente : 3 ;

- les grès subaffleurants, les schistes gréseux: 2;

- les terrasses anciennes: 1.

- Carte de simulation du risque lié à la géomorphologie

L'évaluation du risque peut être faite en fonction des masses déplacées, nous avons donc délimité les secteurs où domine chaque type d'instabilité en leur attribuant un indice de risque, comme suit:

- secteur à éboulements et à glissements de terrain : 5 ; - secteur à solifluxions: 4 ;

- secteur à coulées anciennes et à ravinements: 3 ; - secteur à petites instabilités potentielles : 2 ;

- secteur ne présentant aucune instabilité: 1 .

Cette délimitation en secteurs où domine chaque type d'instabilité nous permet de traduire la carte des indices d'instabilité en carte de simulation du risque lié à la géomorphologie.

\section{- Carte de l'interprétation du risque (Fig. 2)}

Elle résulte de la superposition des cartes de simulation du risque lié à la pente, à la lithologie et à la géomorphologie. La carte de l'interprétation du risque présente dans chaque secteur figuré une combinaison à trois indices définis dans un ordre bien déterminé $\left(\mathrm{I}_{p} \mathrm{I}_{1} \mathrm{I}_{\mathrm{g}}\right)$.

Le triplet $\left(I_{n} I_{1} I_{p}\right)$ situe la part du risque lié à chaque facteur. Prenons par exemple un secteur qui présente le triplet $(3,4,5)$ :
- la pente est favorable à l'instabilité: 3 ;

- le matériau est très sensible aux mouvements de terrain: 4 :

- les risques manifestés ou à craindre sont les glissements de terrain et les éboulements: 5 .

\section{4}

\section{L'évaluation du risque global et les cartes synthétiques}

La transformation des cartes de facteurs en cartes numériques nous permet d'évaluer le risque naturel suivant deux méthodes: la méthode arithmétique et la méthode probabiliste.

\section{Évaluation arithmétique}

Cette méthode a été utilisée par A. Millies-Lacroix (1968). L'action simultanée des trois facteurs de la carte de l'interprétation du risque est représentée par la somme des différents indices dans chaque secteur. L'exemple du triplet I I I précédent c'est-à-dire $(3,4,5)$ fournit une somme de 12 .

\section{- Carte de la sommation du risque}

L'évaluation du risque global par la méthode arithmétique consiste donc à faire la somme des trois indices des triplets figurant dans la carte de l'interprétation du risque $\left(\mathrm{I}_{\mathrm{p}}+\mathrm{I}_{1}+\mathrm{I}_{\mathrm{g}}\right)$. La carte de la sommation du risque présente douze zones où la somme indiciaire donne un maximum de $14(4+5+5)$ et un minimum de $3(1+1+1)$

\section{- Carte des classes du risque (Fig. 3)}

Les douzes zones de la carte de la sommation du risque laissent apparaître une imprécision dans l'appréciation du risque global, ce qui a nécessité une réduction à quatre classes exprimant des niveaux croissant dans la carte des classes de risque, définies selon les fourchettes suivantes: classe 1 , risque faible ou négligeable $(3,4,5)$; classe 2 , risque moyen $(6,7,8)$; classe 3 , risque élevé $(9,10,11)$ et classe 4 , risque très élevé $(12$, $13,14)$

TABLEAUV Degré d'instabilité des terrains de la région de Taounate en fonction de leur nature lithologique. Instability degree of Taounate area formations according to their lithologic nature.

\begin{tabular}{c|c|c|c|c}
$\begin{array}{c}\text { Nature } \\
\text { lithologique }\end{array}$ & $\begin{array}{c}\text { St } \\
\left(\mathrm{km}^{2}\right)\end{array}$ & $\begin{array}{c}\text { Si } \\
\left(\mathrm{km}^{2}\right)\end{array}$ & $\begin{array}{c}\text { Si/St } \\
(\%)\end{array}$ & $\begin{array}{c}\text { Instabilité } \\
\text { prédominante }\end{array}$ \\
\hline Marnes bleues & 19,737 & 3,675 & 37,4 & Solifluxion \\
\hline Marnes schisteuses & 7,9683 & 2,9764 & 8,2 & Goulée ancienne \\
\hline Grès & 12,3954 & 1,0098 & 14,75 & Ravinement de terrain \\
\hline Schistes grèseux & 7,2663 & 1,0708 & 35,92 & Glissement de terrain \\
\hline Molasses & 1,6662 & 0,5153 & 27 & Coulée ancienne
\end{tabular}




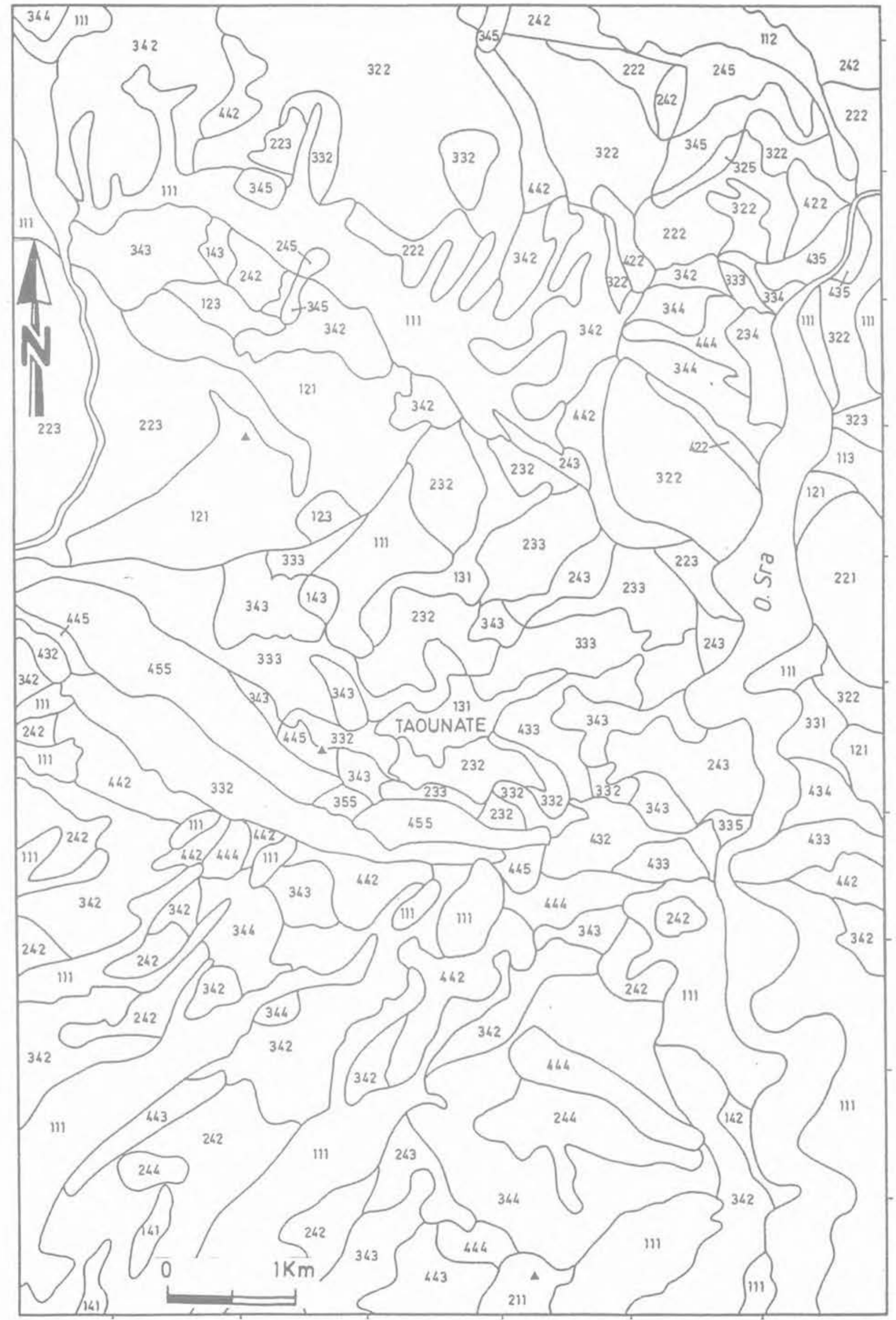

FIG, 2 La carte de l'interprétation du risque. 


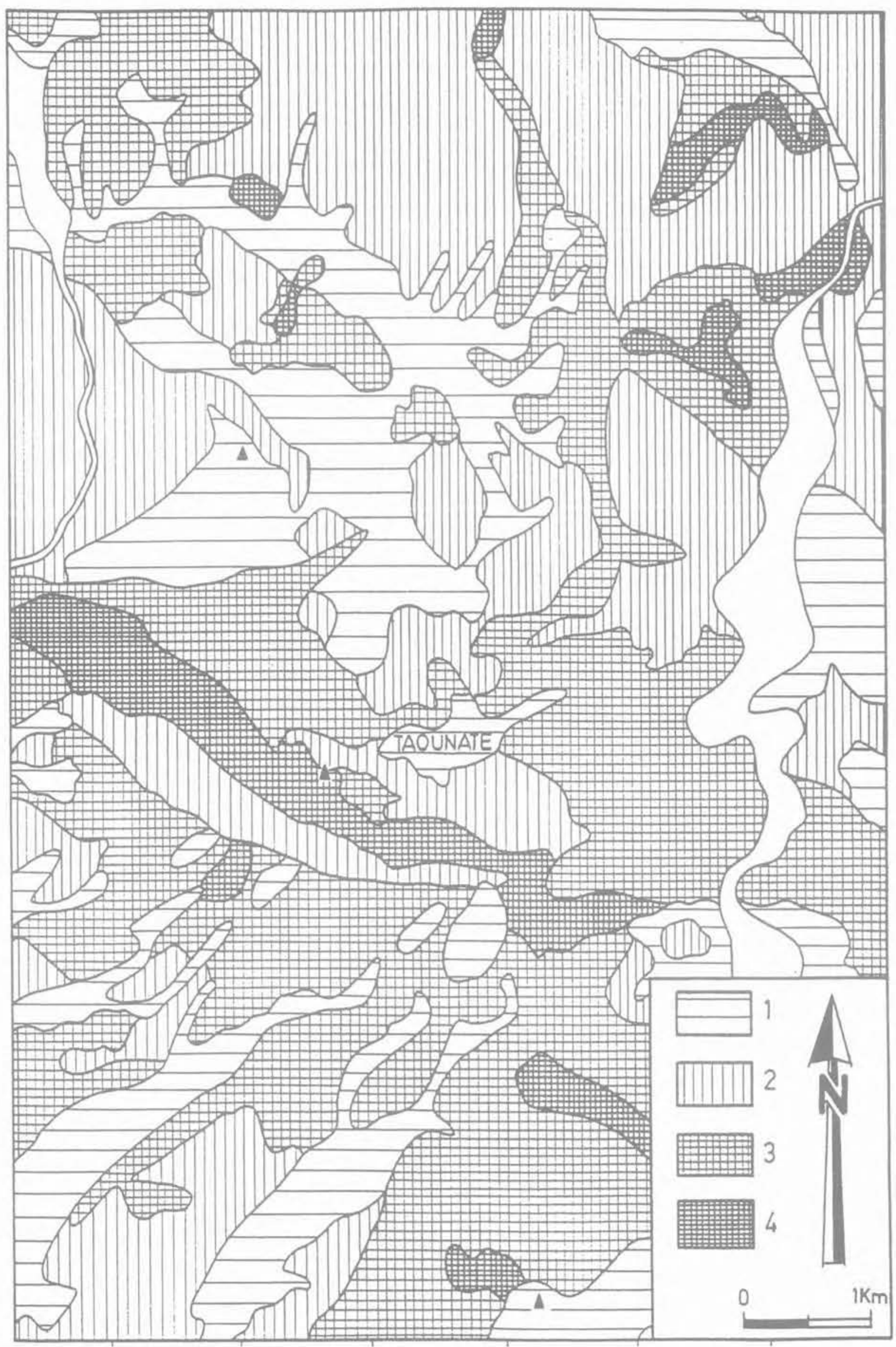

FIG. 3 La carte des classes du risque.

The map of risk classes. 


\section{Évaluation probabiliste}

Nous avons gradué le risque de 0 à 1 et converti les indices du risque en terme de probabilité partielle (Tab. VI).

En conséquence, l'évaluation du risque est estimée égale au produit des probabilités des differents facteurs.

$$
\mathrm{PR}_{\mathrm{R}}(\%)=\mathrm{Pp} \times \mathrm{Pl} \times \mathrm{Pg} \times 100
$$

Pr est la probabilité d'apparition spatiale du risque.

Lorsque tous les facteurs sont à leur maximum d'intensité $\left(\mathrm{P}_{\mathrm{R}}\right)$ est de $100 \%$, valeur pour laquelle la conception du risque est certaine sinon évidente:

$$
\operatorname{PA}(\%)=1 \times 1 \times 1 \times 100=100 \%
$$

Par contre, si les facteurs sont à leur minimum d'íntensité, la probabilité est réduite à $1 \%$.

$$
\mathrm{P}_{\mathrm{H}}(\%)=0,25 \times 0,2 \times 0,2 \times 100=1 \%
$$

- Carte des probabilités d'apparition spatiale du risque

Les facteurs du risque considérés ne varient que dans l'espace et la probabilité d'apparition spatiale écarte donc toute prévision dans le temps.

A partir de la carte de l'interprétation du risque, nous pouvons établir directement la carte de la probabilité d'apparition spatiale du risque qui présente 23 secteurs avec des probabilités allant de 1 à $100 \%$.

\section{- Carte des degrés du risque (Fig.4)}

Nous avons ramené les pourcentages de probabilité de la carte précédente à quatre niveaux du risque croissant: degré du risque faible ou négligeable (1 à 10\%), degré du risque moyen (10 à $30 \%$ ), degré du risque élevé (30 à $50 \%$ ) et degré du risque très élevé (50 à $100 \%$ ).

\section{Comparaison entre les deux méthodes}

La comparaison de l'évaluation arithmétique et de l'évaluation probabiliste porte sur l'action conjointe des facteurs, la lecture de la carte et les rapports des cartes des risques finales avec la carte géologique.

\section{- Action conjointe des facteurs}

La méthode arithmétique conduit à un cumul des intensités des facteurs et on aboutit donc à une appréciation pessimiste. L'évaluation probabiliste met en jeu l'interaction entre les différents facteurs. En effet, il y a un phénomène d'inhibition c'est-à-dire lorsqu'un facteur présente une faible intensité, le risque d'apparition d'une instabilité est moyen, voire faible. On aboutit donc à une appréciation du risque relativement moins subjective.

\section{- Lecture de la carte}

La carte des classes du risque reste interprétative et laisse une part d'imprécision sur l'appréciation du risque présenté par des secteurs d'une même classe ou des secteurs de classes voisines.

TABLEAUVI

Conversion des indices en probabilité.

Conversion of the indices in probability terms.
La carte des degrés du risque au contraire voit cette imprécision levée, car elle exprime le risque par une probabilité d'apparition spatiale, et par conséquent la lecture de la carte directe est facilitée, donc accessible à un large public.

\section{- Rapports de la carte des risques avec la carte géolo- gique}

La carte des classes du risque et la carte des degrés du risque présentent les mêmes tendances dans les secteurs à haut risque et des recoupements quand les secteurs sont classés avec un risque douteux. Cependant les limites de la carte des classes du risque suivent en quelque sorte les contours géologiques, ce qui limite l'intérêt de la démarche arithmétique à l'échelle locale. Cette concordance fait défaut quand on superpose la carte des degrés du risque avec la carte géologique. Il parait possible alors d'adapter cette approche probabiliste de la quantification du risque à d'autres régions présentant des problèmes similaires et ayant des caractéristiques géologiques différentes, et c'est là qui réside tout l'intérêt de l'approche probabiliste par rapport à l'approche arithmétique:

\section{- Méthode choisie pour l'établissement de la carte des risques naturels}

La comparaison des deux méthodes nous a conduit à abandonner la méthode arithmétique et à retenir la méthode probabiliste, car elle met en jeu l'interaction des facteurs et donne une idée relativement précise sur leur action conjointe par la probabilité d'apparition spatiale du risque. Nous utiliserons donc la carte des degrés du risque comme composante fondamentale pour la carte des risques naturels.

\section{5}

\section{La carte des risques naturels liés aux mouvements de terrain}

La carte des degrés du risque exprime la probabilité d'apparition spatiale de mouvements de terrain. Elle ne tient pas compte des zones urbanisées, des routes, etc., bref de la composante humaine surimposée qu'il nous faut introduire dans la carte des risques naturels. En reportant la carte des degrés du risque sur le fond topographique, nous pouvons donc délimiter les zones les plus menacées par les mouvements de terrain. Pour faciliter la lecture et l'utilisation, nous avons employé une représentation cartographique en couleurs conventionnelles du rísque à savoir:

- vert correspond au degré du risque faible ou négligeable, $D_{1}(1$ à $10 \%)$;

- jaune correspond au degré du risque moyen, $D_{2}$ (10 à $30 \%)$;

- orange correspond au degré du risque élevé, $D_{3}$ (30 à $50 \%$ );

- rouge correspond au degré du risque très élevé, $D_{4}$ $(50$ à $100 \%)$.
La pente

\begin{tabular}{|c|c|c|c|c|}
\hline$I_{p}$ & 1 & 2 & 3 & 4 \\
\hline $\mathrm{Pp}$ & 0,25 & 0,5 & 0,75 & 1 \\
\hline
\end{tabular}

La lithologie

La géomorphologie

\begin{tabular}{|c|c|c|c|c|c|}
\hline II & 1 & 2 & 3 & 4 & 5 \\
\hline P1 & 0,2 & 0,4 & 0,6 & 0,8 & 1 \\
\hline
\end{tabular}

\begin{tabular}{|c|c|c|c|c|c|}
\hline $\lg$ & 1 & 2 & 3 & 4 & 5 \\
\hline $\mathrm{Pg}$ & 0,2 & 0,4 & 0,6 & 0,8 & 1 \\
\hline
\end{tabular}




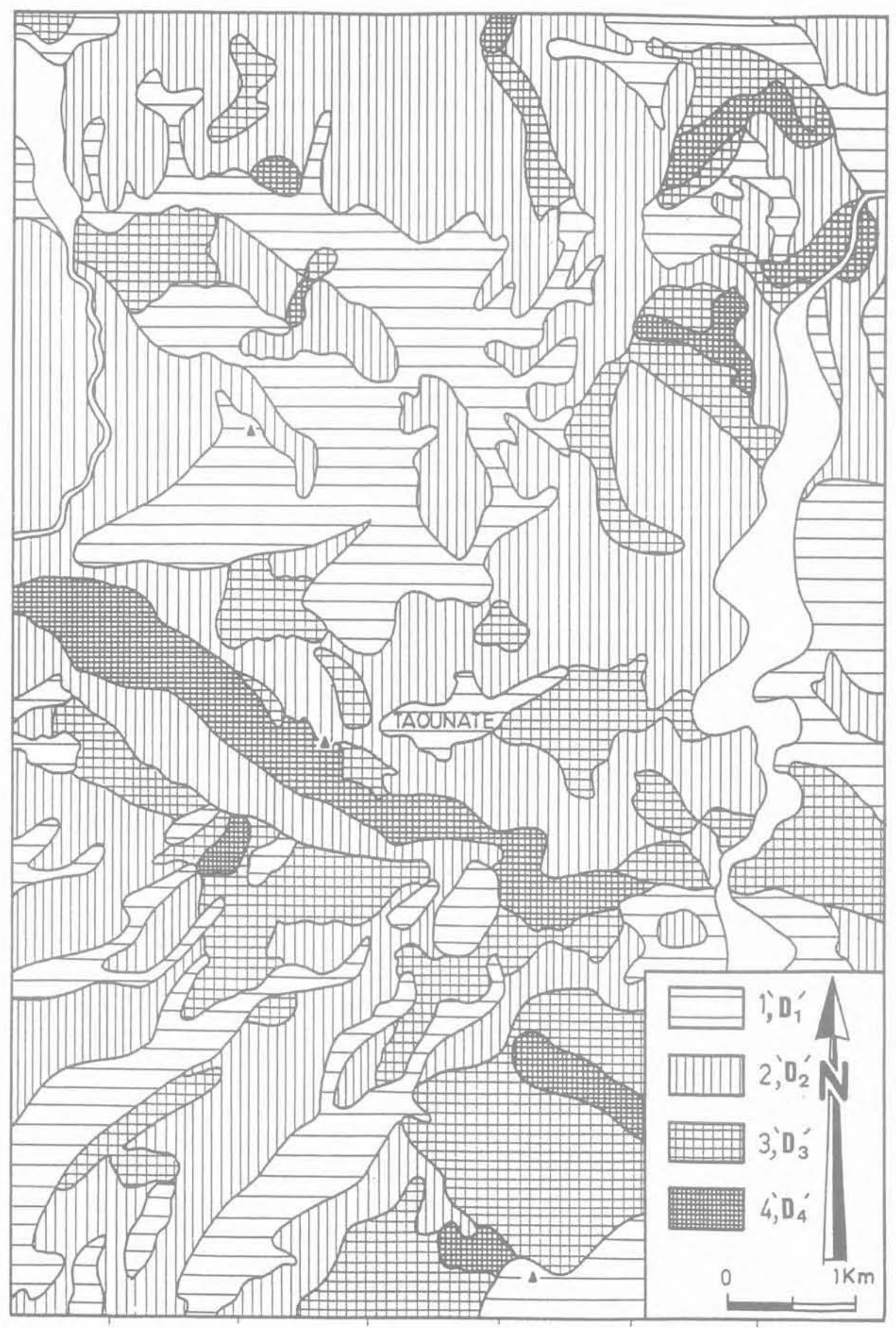

FIG. A La carte des degrés du risque.

The map of the risk degrees. 


\section{Conclusion générale}

La méthode proposée de la cartographie des risques liés aux mouvements de terrain présente une série de cartes allant des cartes de facteurs à la carte des risques naturels. Les étapes intermédiaires sont exposées notamment l'introduction des facteurs du risque (pente, lithologie et géomorphologie), leur quantification et la transformation en cartes numériques. A l'échelle du 1/25000, nous avons considéré les terrains comme le premier paramètre conduisant à l'instabilité.
A une échelle plus petite, nous pouvons faire intervenir les facteurs dynamiques à savoir, le climat, l'homme, la séismicité, etc. A une échelle plus grande, d'autres facteurs prennent de l'importance comme les caractéristiques hydrogéologiques et géotechniques et le suivi des mouvements de terrain en cours, équipés si possible par des instruments de mesures de déplacement.

La carte des risques naturels obtenue par l'approche probabiliste présente une zonation du risque plus fine et plus précise. La représentation du risque en pourcentage facilite la lecture de la carte, donc une utilisation par un large public. A ce titre, elle peut donc être considérée comme la première étape de la prévention contre les mouvements de terrain.

\section{Quelques références bibliographiques*}

Antoine P. (1977) - Réflexions sur la cartographie Zermos et bilan des expériences en cours. Bull. BRGM, III, 1-2, pp. 9-20.

Chazan W. (1973) - Le plan Zermos. Identification des zones exposées aux risques liés aux mouvements du sol et du sous. sol. Symposium national Sol et Sous-sol et sécurité des constructions, Cannes BRGM, 1.

Farès A. (1994) - Essai méthodologique de la cartographie des risques naturels liés aux mouvements de terrain; application à l'aménagement de la ville de Taounate (Rif, Maroc). Thèse Univ. Besançon, $177 \mathrm{p}$.

Humbert M, Jeannette A. (1962) - Les cartes géotechniques: quelques principes de réalisation. $R$. Géogr. Maroc, $n^{\circ} 1-2$, pp. 67-74.

Millies-Lacroix A. (1968) - Les glissements de terrain, présentation d'une carte pré- visionnelle des mouvements de terrain dans le Rif. Mines et géologie, 27, pp. 4554.

Millies-Lacroix A. (1981) - Classification des talus et versants instables. Bull. Liason Labo. Ponts et Chaussées, Spécial X.

Suter G. (1967) - Carte géologique de Taounate-Ain Aicha à 1/50000. Not. et Mém. du Serv. Géol, Maroc, 186 bis. 